gr-qc/0702018

SOGANG-HEP $318 / 07$

\title{
Entropy of a charged black hole in two dimensions without cutoff
}

\author{
Wontae Kim ${ }^{1, a}$, Yong-Wan Kim ${ }^{2, b}$, and Young-Jai Park ${ }^{1, c}$ \\ ${ }^{1}$ Department of Physics and Center for Quantum Spacetime, \\ Sogang University, Seoul 121-742, Korea \\ ${ }^{2}$ National Creative Research Initiative Center for Controlling Optical \\ Chaos, Pai-Chai University, Daejeon 302-735, Korea
}

(May 7, 2021)

\begin{abstract}
By introducing the generalized uncertainty principle (GUP) on quantum density states, we newly obtain a consistent entropy of a scalar field on the (1+1)-dimensional Maxwell-dilaton background without an artificial cutoff in contrast to the results of the brick wall model, which depend on the choice of the Hawking temperature for the extremal case.
\end{abstract}

PACS numbers: 04.70.Dy, 04.60.Kz, 04.62.+v

Keywords: Generalized uncertainty relation; black hole; Maxwelldilaton; entropy.

${ }^{a}$ wtkim@sogang.ac.kr

${ }^{b}$ ywkim@pcu.ac.kr

cyjpark@sogang.ac.kr 


\section{Introduction}

Three decades ago, Bekenstein suggested that the entropy of a black hole is proportional to the area of the horizon through the thermodynamic analogy [1]. Subsequently, Hawking showed that the entropy of the Schwarzschild black hole satisfies exactly the area law by means of Hawking radiation based on the quantum field theory [2]. After their works, 't Hooft investigated the statistical properties of a scalar field outside the horizon of the Schwarzschild black hole by using the brick wall method (BWM) with the Heisenberg uncertainty principle (HUP) [3]. The entropy proportional to the horizon area is obtained, however, a brick wall cutoff, which was introduced to remove the divergence of the density of states, looks unnatural. This method has been used to study the statistical properties of bosonic and fermionic fields in various black holes $[4,5]$, and it is found that the general expression of the black hole entropy consists of the term, which is proportional to the area of the horizon, and the logarithmic and infrared divergent terms.

On the other hand, many efforts have been devoted to the generalized uncertainty relations [6], and its consequences, especially the finite effect on the density of states near horizon. Recently, in Refs. [7, 8, 9], the authors calculated the entropy of various black holes, which is proportional to the horizon area related to the inverse power term of the minimal length, by using the new equation of state density motivated by the generalized uncertainty

principle (GUP) [6], which drastically solves the ultraviolet divergences of the just vicinity near the horizon without any cutoff. It is, however, not clear how this proposal with the GUP works in two dimensions when the horizon is just a point. Up to now, the statistical entropy [10] of the $1+1$ extremal charged black hole was only studied by using the BWM $[13,14]$ with the HUP. Moreover, for the case of a charged black hole, there exists the extremal case for which two horizons coalesce. There are two different choices depending on the Hawking temperature for entropy of the extremal case. One is to take the nonvanishing arbitrary period of temperature in the Euclidean formalism and the corresponding entropy may be a nonzero [15]. The other is the usual vanishing Hawking temperature given by the standard temperature formula and the entropy could be zero.

In this paper, we study the entropy of a scalar field on the background of the two-dimensional charged black hole based on the generalized uncertainty principle. First, we briefly recapitulate the previous results [13] for both 
nonextremal and extremal cases in the BWM with the HUP, which were obtained by using an artificial brick wall cutoff and little mass approximation. But, in this work, we will avoid the difficulty in solving the Klein-Gordon wave equation by using the quantum statistical method. For the nonextremal case the entropy is logarithmic divergent whereas the extremal case gives the vanishing entropy if we take the standard vanishing Hawking temperature. In view of the microcanonical ensemble, however, we shall recast the entropy for the extremal case by counting the number of the states with zero Hawking temperature by using the BWM. This result for the entropy amounts to the same logarithmic-divergent entropy with the nonextremal result. Next, we derive the free energy of a massive scalar field on the background of the twodimensional charged black hole directly by using the new equation of density states motivated by the GUP in the quantum gravity. Then, we calculate the quantum entropy of the black hole via the relation between the free energy and the entropy. As a result, for both nonextremal and extremal cases, we newly obtain the desired same nonzero entropy without any artificial cutoff and little mass approximation in contrast to the BWM case with the HUP.

\section{Scalar field on 1+1 Maxwell-dilaton Back- ground}

Let us now start with the two-dimensional Maxwell-dilaton action induced by the low energy heterotic string theory $[11,12]$, which is given by

$$
S=\frac{1}{2 \pi} \int d^{2} x \sqrt{-g} e^{-2 \phi}\left[R+4(\nabla \phi)^{2}+4 \Lambda^{2}-\frac{1}{4} F^{2}\right],
$$

where $\phi$ is a dilaton field, $\Lambda^{2}$ is cosmological constant, and $F$ is a Maxwell field tensor. The equations of motion under the variations of the metric, dilaton, and gauge fields are given by as follows:

$$
\begin{aligned}
& R_{\mu \nu}-\frac{1}{2} g_{\mu \nu} R+2\left[\nabla_{\mu} \nabla_{\nu} \phi+g_{\mu \nu}\left((\nabla \phi)^{2}-\nabla^{2} \phi-\Lambda^{2}+\frac{1}{16} F^{2}\right)\right]=0, \\
& R+4 \nabla^{2} \phi-4(\nabla \phi)^{2}+4 \Lambda^{2}-\frac{1}{4} F^{2}=0, \\
& \partial_{\nu}\left(\sqrt{-g} e^{-2 \phi} F^{\mu \nu}\right)=0 .
\end{aligned}
$$


In the Schwarzschild gauge, the metric and field tensors are assumed to be

$$
\begin{aligned}
d s^{2} & =-f(r) d t^{2}+\frac{1}{f(r)} d r^{2}, \\
F_{r t} & =F_{r t}(r) .
\end{aligned}
$$

The equations of motion in this gauge with respect to the metric, dilaton, and gauge field yield

$$
\begin{aligned}
& 0=\partial_{r}\left(f \partial_{r} \phi\right)-2 f\left(\partial_{r} \phi\right)^{2}-\frac{1}{4}\left(F_{r t}\right)^{2}+2 \Lambda^{2}, \\
& 0=\partial_{r}^{2} \phi \\
& 0=\partial_{r}\left(F_{r t} e^{-2 \phi}\right) .
\end{aligned}
$$

Simple static solutions with suitable boundary conditions are known as follows:

$$
\begin{aligned}
\phi(r) & =\frac{1}{4} \ln 2-\Lambda r, \\
F_{r t}(r) & =\sqrt{2} Q e^{-2 \Lambda r}, \\
f(r) & =1-\frac{M}{\Lambda} e^{-2 \Lambda r}+\frac{Q^{2}}{4 \Lambda^{2}} e^{-4 \Lambda r} .
\end{aligned}
$$

There are two coordinate singularities $r_{ \pm}$which correspond to the positions of the outer event horizon and the inner Cauchy horizon:

$$
r_{ \pm}=\frac{1}{2 \Lambda} \ln \left[\frac{M}{2 \Lambda} \pm \sqrt{\left(\frac{M}{2 \Lambda}\right)^{2}-\left(\frac{Q}{2 \Lambda}\right)^{2}}\right],
$$

where the Cosmic censorship leads to the condition $M \geq Q$. The Hawking temperature is given by the surface gravity method [10]:

$$
T_{H}=\frac{\Lambda}{2 \pi}\left[1-e^{-2 \Lambda\left(r_{+}-r_{-}\right)}\right],
$$

In this Maxwell-dilaton background, let us first consider a scalar field with mass $m$ under the background (7)-(9), which satisfies the Klein-Gordon equation

$$
\frac{1}{\sqrt{-g}} \partial_{\mu}\left(\sqrt{-g} g^{\mu \nu} \partial_{\nu} \Phi\right)-m^{2} \Phi=0 .
$$


Substituting the wave function $\Phi(r, \theta, t)=e^{-i \omega t} R(r, \theta)$, we find that this Klein-Gordon equation becomes

$$
\frac{d^{2} R}{d r^{2}}+\frac{1}{f} \frac{d f}{d r} \frac{d R}{d r}+\frac{1}{f}\left(\frac{\omega^{2}}{f}-m^{2}\right) R=0 .
$$

By using the Wenzel-Kramers-Brillouin (WKB) approximation [3] with $R \sim \exp [i S(r, \theta)]$, we have

$$
p_{r}^{2}=\frac{1}{f}\left(\frac{\omega^{2}}{f}-m^{2}\right)
$$

where

$$
p_{r}=\frac{d S}{d r} .
$$

On the other hand, we also have the square module momentum

$$
p^{2}=p_{r} p^{r}=g^{r r}{p_{r}}^{2}=f p_{r}{ }^{2}=\frac{\omega^{2}}{f}-m^{2}
$$

with the condition $\omega \geq m \sqrt{f}$.

\section{Brick Wall Models with HUP}

According to the BWM with the HUP, let us briefly recapitulate the previous work [13] in the background of the $1+1$ dimensional charged black hole. However, we would like to avoid the difficulty of solving the wave equation by using the quantum statistical method. The usual position-momentum uncertainty relation followed by the HUP is given by

$$
\Delta x \Delta p \geq \frac{\hbar}{2}
$$

From now on we take the units $G=c=\hbar=k_{B} \equiv 1$. When gravity is ignored, the number of quantum states based on the HUP in the $1+1$ dimension is given by

$$
d n=\frac{d r d p_{r}}{2 \pi}
$$


Then, the number of quantum states with energy less than $\omega$ is given by

$$
\begin{aligned}
n_{O}(\omega) & =\frac{1}{2 \pi} \int d r d p_{r} \\
& =\frac{1}{\pi} \int_{r_{+}+\epsilon}^{L} d r \frac{1}{\sqrt{f}}\left(\frac{\omega^{2}}{f}-m^{2}\right)^{\frac{1}{2}} .
\end{aligned}
$$

Note that $\epsilon$ and $L$ are ultraviolet and infrared regulators, respectively.

On the other hand, for the bosonic case the free energy at inverse temperature $\beta$ is given by

$$
e^{-\beta F}=\prod_{K}\left[1-e^{-\beta \omega_{K}}\right]^{-1},
$$

where $K$ represents the set of quantum numbers. By using Eq. (20), the free energy can be rewritten as

$$
\begin{aligned}
F_{O} & =\frac{1}{\beta} \sum_{K} \ln \left[1-e^{-\beta \omega_{K}}\right] \approx \frac{1}{\beta} \int d n_{O}(\omega) \ln \left[1-e^{-\beta \omega}\right] \\
& =-\int_{\mu \sqrt{f}}^{\infty} d \omega \frac{n_{O}(\omega)}{e^{\beta \omega}-1} \\
& =-\frac{1}{\pi} \int_{r_{+}+\epsilon}^{L} d r \frac{1}{\sqrt{f}} \int_{m \sqrt{f}}^{\infty} d \omega \frac{\left(\frac{\omega^{2}}{f}-m^{2}\right)^{\frac{1}{2}}}{e^{\beta \omega}-1} .
\end{aligned}
$$

Here we have taken the continuum limit in the first line and integrated by parts in the second line.

Now, let us evaluate the entropy for the scalar field, which can be obtained from the free energy (22) at the Hawking temperature. Then, the entropy is

$$
\begin{aligned}
S_{O} & =\left.\beta^{2} \frac{\partial F_{O}}{\partial \beta}\right|_{\beta=\beta_{H}} \\
& =\left.\frac{\beta^{2}}{\pi} \int_{r_{+}+\epsilon}^{L} d r \frac{1}{\sqrt{f}} \int_{m \sqrt{f}}^{\infty} d \omega \frac{\omega e^{\beta \omega}\left(\frac{\omega^{2}}{f}-m^{2}\right)^{\frac{1}{2}}}{\left(e^{\beta \omega}-1\right)^{2}}\right|_{\beta=\beta_{H}},
\end{aligned}
$$

where $\beta_{H}$ is the inverse Hawking temperature. Note that at this stage it is difficult to carry out the analytic integral about $\omega$ because the value of $m \sqrt{f}$ varies depending on $r$ in the wide range $\left(r_{+}+\epsilon, L\right)$. 
For the case of the massless limit, the entropy becomes

$$
\begin{aligned}
S_{O} & =\left.\frac{1}{\beta \pi} \int_{r_{+}+\epsilon}^{L} d r \frac{1}{f} \int_{0}^{\infty} \frac{e^{x} x^{2} d x}{\left(e^{x}-1\right)^{2}}\right|_{\beta=\beta_{H}}, \\
& =\frac{2 \zeta(2)}{\pi \beta_{H}} \int_{r_{+}+\epsilon}^{L} d r \frac{1}{f},
\end{aligned}
$$

where $x=\beta \omega$. Finally, we have the entropy as follows

$$
\begin{aligned}
S_{O} & =\frac{\pi}{6 \Lambda \beta_{H}} \frac{1}{\left(e^{2 \Lambda r_{+}}-e^{2 \Lambda r_{-}}\right)} \times \\
& {\left[e^{2 \Lambda r_{+}} \ln \left(\frac{e^{2 \Lambda L}-e^{2 \Lambda r_{+}}}{e^{2 \Lambda\left(r_{+}+\epsilon\right)}-e^{2 \Lambda r_{+}}}\right)-e^{2 \Lambda r_{-}} \ln \left(\frac{e^{2 \Lambda L}-e^{2 \Lambda r_{-}}}{e^{2 \Lambda\left(r_{+}+\epsilon\right)}-e^{2 \Lambda r_{-}}}\right)\right] . }
\end{aligned}
$$

Furthermore, neglecting the finite part of the entropy for simple consideration, one of us obtained that the most dominant degree of entropy for the non-extremal case is logarithmic divergent as

$$
S_{O} \approx \frac{1}{12} \ln \frac{1}{2 \Lambda \epsilon}
$$

with the cancelation of the infrared divergent $L$-terms, while, for the extremal case just on account of $\beta \rightarrow \infty$ as

$$
S_{O}^{e x t}=\left.\beta^{2} \frac{\partial F_{O}}{\partial \beta}\right|_{\beta \rightarrow \infty}=0 \quad\left(r_{+}=r_{-}\right)
$$

On the other hand, for the extremal case from a zero temperature quantummechanical system around the black hole a similar form of the entropy was obtained as $S_{O}^{e x t} \approx \ln \frac{1}{2 \Lambda \epsilon}$. [13] However, these strange results including logarithmic divergence will be drastically changed due to the GUP effect in the next section.

\section{Entropy with Generalized Uncertainty Prin- ciple}

Recently, many efforts have been devoted to the generalized uncertainty relation [6] given by

$$
\Delta x \Delta p \geq \frac{1}{2}\left(1+\lambda(\Delta p)^{2}\right)
$$


Then, since one can easily get $\Delta x \geq \sqrt{\lambda}$, which gives the minimal length, it can be defined to be the thickness of the thin-layer near horizon, which naturally plays a role of the brick wall cutoff. Furthermore, based on the generalized uncertainty relation, the volume of a phase cell in the $1+1$ dimensions is changed from $(2 \pi)$ into

$$
2 \pi\left(1+\lambda p^{2}\right)
$$

where $p^{2}=p^{r} p_{r}$.

From Eq. (29), the number of quantum states with energy less than $\omega$ is given by

$$
\begin{aligned}
n_{I}(\omega) & =\frac{1}{2 \pi} \int d r d p_{r} \frac{1}{1+\lambda\left(\frac{\omega^{2}}{f}-m^{2}\right)} \\
& =\frac{1}{\pi} \int d r \frac{1}{\sqrt{f}} \frac{\left(\frac{\omega^{2}}{f}-m^{2}\right)^{\frac{1}{2}}}{1+\lambda\left(\frac{\omega^{2}}{f}-m^{2}\right)} .
\end{aligned}
$$

Note that it is convergent at the horizon without any artificial cutoff due to the existence of the suppressing $\lambda$ term in the denominator induced from the GUP. Then, by using Eq. (30), the free energy at the Hawking temperature can be obtained as

$$
\begin{aligned}
F_{I} & =-\int_{m \sqrt{f}}^{\infty} d \omega \frac{n_{I}(\omega)}{e^{\beta \omega}-1} \\
& =-\frac{1}{\pi} \int d r \frac{1}{\sqrt{f}} \int_{m \sqrt{f}}^{\infty} d \omega \frac{\left(\frac{\omega^{2}}{f}-m^{2}\right)^{\frac{1}{2}}}{\left(e^{\beta \omega}-1\right)\left[1+\lambda\left(\frac{\omega^{2}}{f}-m^{2}\right)\right]}
\end{aligned}
$$

From this free energy, the entropy for the massive scalar field is given by

$$
\begin{aligned}
S_{I} & =\left.\beta^{2} \frac{\partial F_{I}}{\partial \beta}\right|_{\beta=\beta_{H}} \\
& =\left.\frac{\beta^{2}}{\pi} \int d r \frac{1}{\sqrt{f}} \int_{m \sqrt{f}}^{\infty} d \omega \frac{\omega e^{\beta \omega}\left(\frac{\omega^{2}}{f}-m^{2}\right)^{\frac{1}{2}}}{\left(e^{\beta} \omega-1\right)^{2}\left(1+\lambda\left(\frac{\omega^{2}}{f}-m^{2}\right)\right)}\right|_{\beta=\beta_{H}} .
\end{aligned}
$$

Since $f \rightarrow 0$ near the event horizon, i.e., in the range of $\left(r_{+}, r_{+}+\epsilon\right)$, then, without little mass approximation, the entropy is reduced to 


$$
S_{I}=\left.\frac{1}{\pi} \int d r \frac{1}{\sqrt{f}} \int_{0}^{\infty} d x \frac{f^{-\frac{1}{2}} \beta^{-1} x^{2}}{\left(1-e^{-x}\right)\left(e^{x}-1\right)\left(1+\frac{\lambda}{\beta^{2} f} x^{2}\right)}\right|_{\beta=\beta_{H}},
$$

where $x=\beta \omega$.

On the other hand, we are only interested in the contribution from the just vicinity near the horizon, $\left(r_{+}, r_{+}+\epsilon\right)$, which corresponds to a proper distance of order of the minimal length, $\sqrt{\lambda}$. This is because the entropy closes to the upper bound only in this vicinity, which it is just the vicinity neglected by BWM. We have

$$
\sqrt{\lambda}=\int_{r_{+}}^{r_{+}+\epsilon} \frac{d r}{\sqrt{f(r)}}=\int_{r_{+}}^{r_{+}+\epsilon} \frac{d r}{\sqrt{2 \kappa\left(r-r_{+}\right)}}=\sqrt{\frac{2 \epsilon}{\kappa}},
$$

where $\kappa$ is the surface gravity at the horizon of black hole and it is identified as $\kappa=2 \pi \beta$.

Now, let us rewrite Eq. (33) as

$$
S_{I}=\frac{1}{\pi \sqrt{\lambda}} \int_{r_{+}}^{r_{+}+\epsilon} d r \frac{1}{\sqrt{f}} \Sigma_{I},
$$

where $\Sigma_{I}$ is given by

$$
\Sigma_{I}=\int_{0}^{\infty} d X \frac{a^{2} X^{2}}{\left(e^{\frac{a}{2} X}-e^{-\frac{a}{2} X}\right)^{2}\left(1+X^{2}\right)}
$$

with $x=\beta \sqrt{\frac{f}{\lambda}} X \equiv a X$. Note that the entropy is $\beta$ independent at this stage. As $r \rightarrow r_{+}, a$ goes to 0 . Since we are interested in the contributions from just the vicinity of the horizon, the integral equation (36) can be easily solved as follows

$$
\Sigma_{I}=\int_{0}^{\infty} \frac{d X}{1+X^{2}}=\frac{\pi}{2} .
$$

Finally, when $r \rightarrow r_{+}$, we newly get a $\beta$-independent same entropy as follows

$$
S_{I} \approx \frac{1}{\pi \sqrt{\lambda}} \cdot \sqrt{\lambda} \cdot \frac{\pi}{2}=\frac{1}{2}
$$

for both nonextremal and extremal black holes. Note that in contrast to the case of the BWM there is no divergence within the just vicinity near 
the horizon due to the effect of the generalized uncertainty relation on the quantum states.

Now, it seems appropriate to comment on the entropy (38). First, for the extremal case $\left(r_{H}=r_{+}=r_{-}\right)$we could also obtain the entropy from a zero temperature quantum-mechanical system around the black hole with the GUP following the standard formula for extracting entropy from the partition function such as

$$
S_{O}^{e x t}=\left.\left(1-\beta \frac{\partial}{\partial \beta}\right) \ln Z\right|_{\beta=\infty} .
$$

By using the density of stats $D_{I}(\omega)=\frac{d n_{I}(\omega)}{d \omega}$, which is obtained from Eq. (30), the thermodynamic partition function can be obtained as

$$
\begin{aligned}
\ln Z & =-\int d \omega D_{I}(\omega) \ln \left[1-e^{-\beta \omega}\right] \\
& =\frac{1}{\pi} \int_{r_{H}}^{r_{H}+\epsilon} d r \frac{1}{\sqrt{f}} \int_{m \sqrt{f}}^{\infty} d \omega \frac{\beta\left(\frac{\omega^{2}}{f}-m^{2}\right)^{\frac{1}{2}}}{\left(e^{\beta \omega}-1\right)\left[1+\lambda\left(\frac{\omega^{2}}{f}-m^{2}\right)\right]}
\end{aligned}
$$

Then, from the above partition function we can easily obtain the same value of the entropy as that in Eq. (38). This means that by considering the GUP the entropy does not depend on the choice of Hawking temperature in contrast to the BWM. Second, since the entropy is proportional to the inverse power term of the minimal length $\sqrt{\lambda}$, which depends on the $(d+1)$ dimensions as $S \propto(\sqrt{\lambda})^{-(d-1)}[7,8,9]$, we have consistently obtained the $\sqrt{\lambda}$ independent desired entropy in the $(1+1)$-dimensions.

In summary, we have investigated the massive scalar field within the just vicinity near the horizon of a static black hole in the background of the charged black hole in the $1+1$ dimensions by using the generalized uncertainty principle. In contrast to the previous result of the BWM $[13,14]$, we have newly obtained the proper entropy without any artificial cutoff and little mass approximation.

\section{Acknowledgments}

We would like to thank M.S. Yoon for useful discussions. This work is supported by the Science Research Center Program of the Korea Science and 
Engineering Foundation through the Center for Quantum Spacetime of Sogang University with grant number R11-2005-021.

\section{References}

[1] J. D. Bekenstein, Lett. Nuovo Cimento 4, 737 (1972); Phys. Rev. D 7, 2333 (1973); Phys. Rev. D 9, 3292 (1974).

[2] S. W. Hawking, Commun. Math. Phys. 43, 199 (1975).

[3] G. 't Hooft, Nucl. Phys. B 256, 727 (1985).

[4] A. Ghosh and P. Mitra, Phys. Rev. Lett. 73, 2521 (1994); J-G. Demers, R. Lafrance, and R. C. Myers, Phys. Rev. D 52, 2245 (1995); S. P. de Alwis and N. Ohta, Phys. Rev. D 52, 3529 (1995); R.B. Mann and S.N. Solodukhin, Phys. Rev. D 54, 3932 (1996); R.G. Cai and Y.-Z. Zhang, Mod. Phys. Lett. A 11, 2027 (1996); V.P. Frolov, D.V. Fursaev, Phys. Rev. D 56, 2212 (1997); J. Jing and M.-L. Yan, Phys. Rev. D 60, 084015 (1999); E. Winstanley, Phys. Rev. D 63, 084013 (2001); A.J.M. Medved, Class. Quantum Grav. 19, 405 (2002); M. Kenmoku, K. Ishimoto, K.K. Nandi, and K. Shigemoto, Phys. Rev. D 73, 064004 (2006).

[5] S. W. Kim, W. T. Kim, Y. J. Park, and H. Shin, Phys. Lett. B 392, 311 (1997); J.-W. Ho, W. T. Kim, Y.-J. Park, and H.-J. Shin, Class. Quantum Grav. 14, 2617 (1997); S.P. Kim, S.K. Kim, K.-S. Soh, and J.H. Yee, Phys. Rev. D 55, 2159 (1997); W. T. Kim, J.J. Oh, and Y. J. Park, Phys. Lett. B 512, 131 (2002).

[6] A. Kempf, G. Mangano and R. B. Mann, Phys. Rev. D 52, 1108 (1995); L. J. Garay, Int. J. Mod. Phys. A 10, 145 (1995); S. K. Rama, Phys. Lett. B 519, 103 (2001); R. J. Adler, P. Chen and D. I. Santiago, Gen. Rel. Grav. 33, 2101 (2002); L. N. Chang, D. Minic, N. Okamura and T. Takeuchi, Phys. Rev. D 65, 125028 (2002); Y.S. Myung, Y.-W. Kim, and Y.-J. Park, Phys. Lett. B 645, 393 (2007).

[7] X. Li, Phys. Lett. B 540, 9 (2002).

[8] W. B. Liu, Chin. Phys. Lett. 20, 440 (2003); C.-Z. Liu, Int. J. Theor. Phys. 44, 567 (2005). 
[9] W. Kim, Y.-W. Kim, and Y. J. Park, J. Korean. Phys. Soc. 46, 1360 (2006); W. Kim, Y.-W. Kim, and Y.-J. Park, Phys. Rev. D 74, 100401 (2006).

[10] J. Maldacena and A. Strominger, JHEP 02, 014 (1998).

[11] M. McGuigan, C. Nappi, and S. Yost, Nucl. Phys. B 375, 421 (1992); C. Nappi and A. Pasquinucci, Mod. Phys. Lett. A 7, 3337 (1992).

[12] S. Nojiri and S. P. Odintsov, Phys. Rev. D 59, 044003 (1999).

[13] H. Lee, S.-W. Kim, and W.T. Kim, Phys. Rev. D 54, 6559 (1996).

[14] B. Wang and R.-K. Su, Phys. Rev. D 59, 104006 (1999).

[15] S. W. Hawking, G. Horowitz, and S. Ross, Phys. Rev. D 51, 4302 (1995); A. Ghosh and P. Mitra, Phys. Lett. B 357, 295 (1995). 